\title{
Inside a Beehive: The Multiple Merging Processes in the Galaxy Cluster Abell 2142*
}

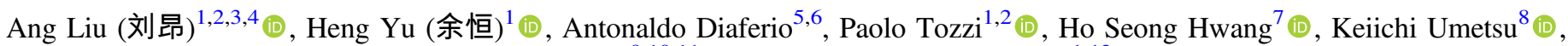 \\ Nobuhiro Okabe ${ }^{9,10,11}$ (D), and Li-Lan Yang (杨里岗) ${ }^{1,12}$ (D) \\ ${ }^{1}$ Department of Astronomy, Beijing Normal University, Beijing, 100875, People’s Republic of China; yuheng@bnu.edu.cn, liuang@arcetri.astro.it \\ 2 INAF Osservatorio Astrofisico di Arcetri, Largo E. Fermi, I-50122 Firenze, Italy \\ ${ }^{3}$ Department of Physics, Sapienza University of Rome, I-00185, Rome, Italy \\ ${ }^{4}$ Department of Physics, University of Rome Tor Vergata, I-00133, Rome, Italy \\ ${ }^{5}$ Dipartimento di Fisica, Università di Torino, Via P. Giuria 1, I-10125 Torino, Italy \\ ${ }^{6}$ Istituto Nazionale di Fisica Nucleare (INFN), Sezione di Torino, Via P. Giuria 1, I-10125 Torino, Italy \\ ${ }^{7}$ Quantum Universe Center, Korea Institute for Advanced Study, 85 Hoegiro, Dongdaemun-gu, Seoul 02455, Republic of Korea \\ ${ }^{8}$ Institute of Astronomy and Astrophysics, Academia Sinica, P.O. Box 23-141, Taipei 10617, Taiwan \\ ${ }^{9}$ Department of Physical Science, Hiroshima University, 1-3-1, Kagamiyama, Higashi-Hiroshima, Hiroshima 739-8526, Japan \\ ${ }^{10}$ Hiroshima Astrophysical Science Center, Hiroshima University, 1-3-1, Kagamiyama, Higashi-Hiroshima, Hiroshima 739-8526, Japan \\ ${ }^{11}$ Core Research for Energetic Universe, Hiroshima University, 1-3-1, Kagamiyama, Higashi-Hiroshima, Hiroshima 739-8526, Japan \\ ${ }^{12}$ School of Physics and Technology, Wuhan University, Wuhan, 430072, People's Republic of China \\ Received 2018 March 5; revised 2018 June 28; accepted 2018 June 29; published 2018 August 14
}

\begin{abstract}
To investigate the dynamics of the galaxy cluster A2142, we compile an extended catalog of 2239 spectroscopic redshifts of sources, including 237 newly measured redshifts, within 30 arcmin from the cluster center. With the $\sigma$-plateau algorithm from the caustic method, we identify 868 members and a number of substructures in the galaxy distribution both in the outskirts, out to $\sim 3.5 \mathrm{Mpc}$ from the cluster center, and in the central region. In the outskirts, one substructure overlaps a falling clump of gas previously identified in the X-ray band. These substructures suggest the presence of multiple minor mergers, which are responsible for the complex dynamics of A2142, and the absence of recent or ongoing major mergers. We show that the distribution of the galaxies in the cluster core and in several substructures is consistent with the mass distribution inferred from the weak-lensing signal. Moreover, we use spatially resolved X-ray spectroscopy to measure the redshift of different regions of the intracluster medium within $\sim 3$ arcmin from the cluster center. We find a ring of gas near the two X-ray cold fronts identified in previous analyses and measure a velocity of this ring of $810 \pm 330 \mathrm{~km} \mathrm{~s}^{-1}$ larger than the cluster mean velocity. Our analysis suggests the presence of another ring surrounding the core, whose velocity is $660 \pm 300 \mathrm{~km} \mathrm{~s}^{-1}$ larger than the cluster velocity. These X-ray features are not associated with any optical substructures, and support the core-sloshing scenario suggested in previous work.
\end{abstract}

Key words: galaxies: clusters: general - galaxies: clusters: individual (Abell 2142) - galaxies: clusters: intracluster medium

Supporting material: machine-readable table

\section{Introduction}

Galaxy clusters link the evolution of the large-scale structure to the astrophysical processes on smaller scales, and the study of their assembling is thus crucial for understanding the hierarchical evolution of the universe. The commonly accepted scenario is that clusters form and evolve via accretion and merging of smaller halos. This scenario is suggested by many dynamical features observed in clusters: substructures in the galaxy distribution (Geller \& Beers 1982; Wen \& Han 2013; Guennou et al. 2014; Girardi et al. 2015; Zarattini et al. 2016); apparent global rotation of clusters (Hwang \& Lee 2007; Manolopoulou \& Plionis 2017); clumpy distributions (Gutierrez \& Krawczynski 2005; Parekh et al. 2015; Yu et al. 2016; Parekh et al. 2017) and bow shocks (Markevitch et al. 2002, 2005) in the intracluster medium (ICM) observed in X-rays; the elongated or peculiar distributions of radio emission (Feretti et al. 2012; Govoni et al. 2012; Riseley et al. 2017; Rajpurohit et al. 2018); and

\footnotetext{
* We dedicate this paper to the late Bepi Tormen, our beloved friend and colleague whose enthusiastic and intense work on gravitational dynamics largely contributed to our current understanding of the formation and evolution of galaxy clusters.
}

the substructure distribution of the dark matter, inferred from gravitational lensing observations (Okabe \& Umetsu 2008; Okabe et al. 2014; Grillo et al. 2015; Caminha et al. 2017). In addition, "cold fronts" are frequently observed in X-ray images of clusters (Markevitch et al. 2000; Sanders et al. 2005, 2016; Ichinohe et al. 2017), including some regular and relaxed clusters (Mazzotta et al. 2001; Clarke et al. 2004). Cold fronts are X-ray surface brightness edges with an approximately continuous pressure profile across the density discontinuity, at odds with the large pressure jump of shock fronts.

The massive cluster A2142 is one of the most representative clusters with cold fronts. Its Chandra image exhibits an elongated X-ray morphology, and two prominent cold fronts in the opposite directions along the longest axis (Markevitch et al. 2000). The scenario of A2142, suggested by these observational features, was at first envisioned as a pericentric merging of two subclusters, with the two cold fronts delineating the subcluster cores that have survived the merging. This hypothesis was dubbed the "remnant core" scenario (Markevitch et al. 2000), and is appropriate for merging clusters with prominent signatures of recent or ongoing mergers. However, A2142 shows an almost regular 
morphology and appears relaxed at large radii, unlike $1 \mathrm{E}$ 0657-56 (Markevitch et al. 2002), A520 (Govoni et al. 2001; Markevitch et al. 2005; Deshev et al. 2017), and other clusters with cold fronts, which clearly appear unrelaxed. Therefore, Tittley \& Henriksen (2005), Markevitch \& Vikhlinin (2007), and Owers et al. (2011) proposed an alternative model, where the observed cold fronts derive from a sloshing cool core (Markevitch et al. 2001; Churazov et al. 2003).

More recently, another cold front in A2142, at about $1 \mathrm{Mpc}$ from the center to the southeast, was discovered by Rossetti et al. (2013) with XMM-Newton observations, showing that the sloshing in A2142 is not confined to the core, but extends to much larger scales. In addition, both XMM-Newton (Eckert et al. 2014) and Chandra (Eckert et al. 2017) detected a falling clump of hot gas in the outskirts, suggesting that the merging process is still ongoing. Two giant radio halos involved with the sloshing of the cluster core were also revealed by LOFAR and VLA observations (Venturi et al. 2017).

Additional information on the complex dynamics of A2142 derives from the optical band. The presence of substructures in the galaxy distribution was first pointed out by Oegerle et al. (1995) with 103 spectroscopically confirmed galaxies. Based on the spectroscopic redshifts of 956 member galaxies, Owers et al. (2011) concluded that some earlier minor mergers could have induced the sloshing of the core in A2142. More recently, Einasto et al. (2018) suggested that A2142 formed through past and present mergers of smaller groups, determining the complex radio and X-ray structure observed in this cluster.

All these increasingly rich observational data in different bands provide relevant details of the dynamics of A2142, which, when combined, can further clarify the scenario of the formation of A2142. Here, we provide a step forward in this direction by combining the information provided by the optical and X-ray spectroscopy and by the dark matter distribution reconstructed from weak-lensing data. The three methods are complementary, and their combination helps to better understand the assembly history of the cluster and to understand the possible systematic errors of each method (see, e.g., Hwang et al. 2014 and $\mathrm{Yu}$ et al. 2016).

The hierarchical tree method based on optical spectroscopy for the investigation of substructures in galaxy clusters was introduced by Serna \& Gerbal (1996). Diaferio (1999) and Serra et al. (2011) improved this approach by developing the $\sigma$-plateau algorithm for the automatic identification of a threshold to trim the hierarchical tree and identify the cluster substructures. The $\sigma$-plateau algorithm is part of the caustic method (Diaferio \& Geller 1997; Diaferio 1999) that estimates the mass profiles of galaxy clusters out to regions well beyond the virial radius (Serra et al. 2011) and identifies the galaxies members of the cluster (Serra \& Diaferio 2013). Yu et al. (2015) investigated in detail the performance of the $\sigma$-plateau algorithm to identify the substructures in the distribution of the cluster galaxies. Substructure properties, including their redshift, velocity dispersion, and morphology, can provide relevant information on the dynamics of the cluster.

In addition to the optical information, fitting the position of the iron $K_{\alpha}$ line in the X-ray spectrum coming from a defined region of the ICM provides an accurate measure of its redshift (Dupke \& Bregman 2001a, 2001b, 2006; Liu et al. 2015). By measuring the redshift of different ICM regions, one can infer a map of the radial velocities of the ICM, which again provides constraints on the modeling of the ICM dynamics: with this technique, Liu et al. (2016) identified the presence of significant bulk motions in the ICM of A2142 at a $3 \sigma$ confidence level.

The optical and spatially resolved X-ray spectroscopy were successfully combined for the first time by Yu et al. (2016) for the cluster A85, to unveil the origin of its complex accretion process (see also Song et al. 2017 for A2199). Here, we apply this new strategy to investigate the dynamics of A2142.

The paper is organized as follows. In Section 2, we describe our new and extended optical spectroscopic catalog. In Section 3, we identify the substructures in the galaxy distribution. In Section 4, we describe the spatially resolved ICM redshift measurements. In Section 5, we present the method and results of the weak-lensing analysis, and in Section 6, we infer the dynamical state of A2142 by combining the information from our analyses of all the data. We conclude in Section 7. Throughout this paper, we adopt the 7 year WMAP cosmology, with $\Omega_{\mathrm{m}}=0.272, \Omega_{\Lambda}=0.728$, and $H_{0}=$ $70.4 \mathrm{~km} \mathrm{~s}^{-1} \mathrm{Mpc}^{-1}$ (Komatsu et al. 2011). All the errors we mention are at the $1 \sigma$ confidence level.

\section{Optical Spectroscopic Sample}

We compile the currently largest catalog of 2239 spectroscopic redshifts in the field of view of A2142. Our catalog covers an area of $1^{\circ} \times 1^{\circ}$ around the cluster center, corresponding to an area $7 \times 7 \mathrm{Mpc}^{2}$ at the cluster redshift $z=0.09$; the redshifts in our catalog are in the range $[0.01,0.6]$. Our catalog includes 1270 redshifts from the catalog of Owers et al. (2011) that are not in the SDSS DR13 (Albareti et al. 2017); Owers et al.'s full catalog lists 1635 redshifts in the range $z=[0.0088,3.8]$. In our sample, we include 731 additional redshifts from SDSS catalog and one redshift from Oegerle et al. (1995). In 2014 June, to secure more redshifts, we made additional spectroscopic observations with the 300 fiber Hectospec multi-object spectrograph on the MMT $6.5 \mathrm{~m}$ telescope (Fabricant et al. 2005). To obtain a high, uniform spectroscopic completeness in the cluster region, we weighted the targets according to their r-band apparent magnitudes independently of colors. We used the 270 line $\mathrm{mm}^{-1}$ grating for Hectospec observations, which gives a dispersion of $1.2 \AA$ pixel $^{-1}$ and a resolution of $\sim 6 \AA$. The resulting spectra cover the wavelength range 3650-9150 $\mathrm{\AA}$. We observed one field with $\sim 250$ target fibers for $3 \times 20$ minute exposures. The spectra were reduced with the Mink et al. (2007) pipeline, and were cross-correlated with template spectra to determine the redshifts using RVSAO (Kurtz \& Mink 1998). We visually inspected all the spectra, and assigned a quality flag to the spectral fits with "Q" for high-quality redshifts, "?" for marginal cases, and " $\mathrm{X}$ " for poor fits. We then use only the spectra with reliable redshift measurements (i.e., "Q"). In total, we obtained 237 additional redshifts in the field.

Owers et al. (2011), Geller et al. (2016), and Geller et al. (2014) showed that there is basically no systematic bias between MMT and SDSS sources; therefore, it is appropriate to merge the two data sets in the same catalog. As mentioned above, the catalog of Owers et al. (2011) includes redshifts that also appear in the SDSS catalog: we always choose the SDSS measures in this case, because their errors are smaller.

In Figure 1, we show the spectroscopic completeness for $m_{\mathrm{r}, \text { Petro, } 0} \leqslant 20.5$. The two-dimensional completeness map is in $9 \times 9$ pixels for the $42^{\prime} \times 42^{\prime}$ field of view. The overall 


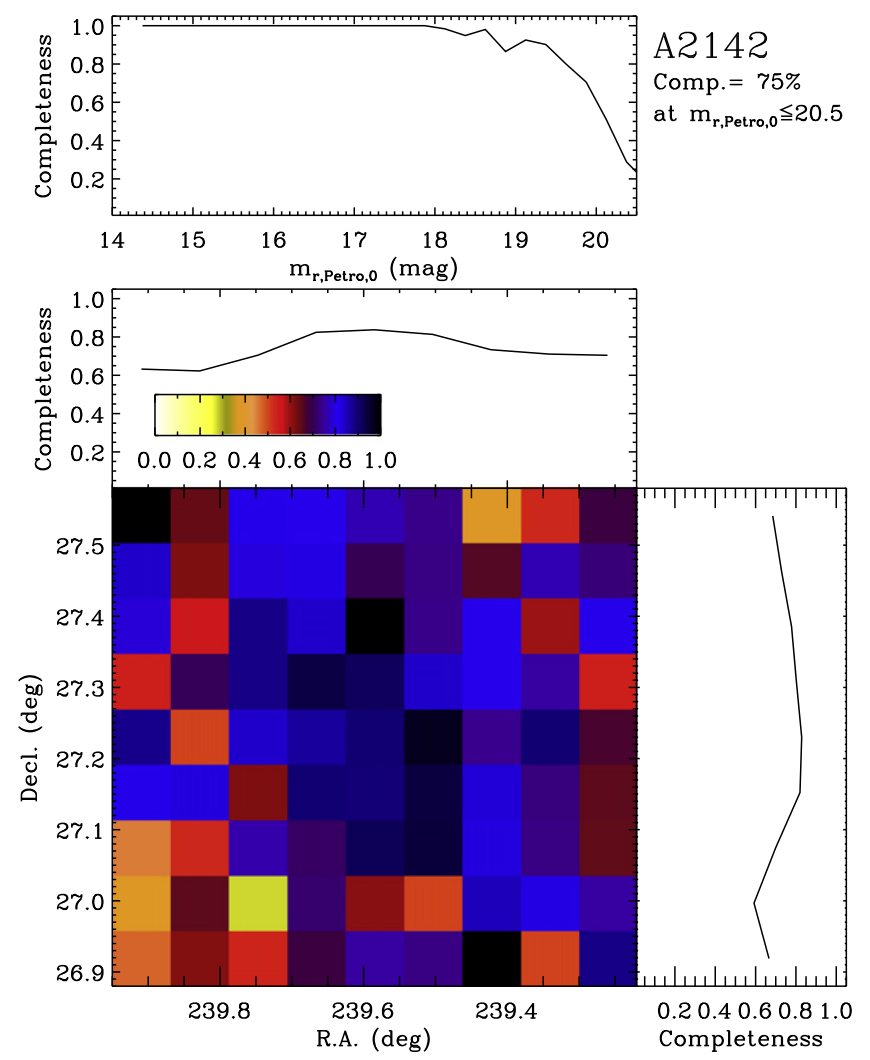

Figure 1. Spectroscopic completeness in the field of A2142.

completeness throughout this field is $75 \%$, with a small spatial variation.

A sample of the redshift catalog is given in Table 1. For each galaxy, the table contains the SDSS ObjID, right ascension (R. A.), declination (decl.), r-band Petrosian magnitude with Galactic extinction correction from SDSS, the redshift ( $z)$, the uncertainty in $z$, and the spectrum and redshift source. The full version of the table is available in the online journal.

Our catalog contains 604 redshifts in addition to the Owers et al. (2011) catalog. Figure 2 shows the distribution of the redshifts in our catalog. There are 1117 redshifts in the range $z=[0.07,0.11], 63$ of which were not in the Owers et al. (2011) catalog. The $3 \sigma$ clipping procedure (Yahil \& Vidal 1977) removes 50 galaxies and thus leaves 1067 galaxies as possible cluster members. The mean redshift and the redshift dispersion of these 1067 galaxies are $0.0901 \pm 0.0001$ and 0.0040 , respectively.

\section{Substructures in the Galaxy Distribution}

For a quantitative investigation of the distribution of galaxies, we adopt the $\sigma$-plateau algorithm. To set up a familiar framework to our results, we also provide an analysis based on the Dressler-Shectman (DS) test, which is more commonly used in the literature.

\subsection{Results from the $\sigma$-plateau Algorithm}

The $\sigma$ plateau algorithm, implemented within the caustic method (Diaferio \& Geller 1997; Diaferio 1999; Serra et al. 2011), is based on optical spectroscopic data and provides an extremely efficient procedure to identify both the cluster members (Serra \& Diaferio 2013) and the cluster substructures (Yu et al. 2015). Unlike the DS method, which only suggests the presence of substructures but does not unambiguously identify them, the $\sigma$-plateau algorithm returns a list of the individual substructures and their members.

The method consists of a classical approach to cluster analysis for grouping sets of objects with similar properties. The caustic method groups the galaxies in the field of view in a binary tree according to an estimate of their pairwise binding energy, derived from the projected separation and the line-ofsight velocity difference of each galaxy pair.

The main branch of the binary tree is the path determined by the nodes of the tree which contain the largest number of galaxies, or leaves, at each bifurcation. The velocity dispersion $\sigma_{i}$ of the leaves of each node $i$ decreases, on average, when walking along the main branch from the root to the leaves.

When the binary tree is built with the galaxies in a field of view containing a galaxy cluster (see Figure 3 for our redshift catalog of A2142), the velocity dispersion $\sigma_{i}$ along the main branch settles onto a $\sigma$ plateau in between two nodes, as shown in Figure 4, where we plot the velocity dispersion $\sigma_{i}$ as a function of the main branch node of A2142. This plateau originates from the quasi-dynamical equilibrium of the cluster: the velocity dispersions $\sigma_{i}$ of the nodes closer to the root (on the left of Figure 4) are larger than the plateau, because these nodes contain a large fraction of galaxies that are not cluster members; the velocity dispersions of the nodes closer to the leaves (on the right of Figure 4) are smaller than the plateau, because at this level the binary tree splits the cluster into its dynamically distinct substructures.

The two boundary nodes of the plateau thus identify two thresholds that are used to cut the tree at two levels: the threshold closer to the root identifies the cluster members; the threshold closer to the leaves identifies the cluster substructures (see Serra \& Diaferio 2013; Yu et al. 2015, for further details). All the systems with at least 6 galaxies below this second threshold enter our list of substructures. Here, as the minimum number of substructure members, we adopt 6 galaxies, rather than 10 galaxies as chosen by $\mathrm{Yu}$ et al. (2015), which appears to be too severe and can exclude real, albeit poor, substructures.

In the plot of the velocity dispersion along the main branch of the binary tree of A2142 shown in Figure 4 we can see three plateaus, rather than one: this feature is typical of clusters with complex dynamics (Yu et al. 2015). The left node, shown by the open red triangle, of the first plateau at $\sim 950 \mathrm{~km} \mathrm{~s}^{-1}$, is the threshold that identifies the cluster. The left node, shown by the open red square, of the second plateau at $\sim 870 \mathrm{~km} \mathrm{~s}^{-1}$, is the threshold that identifies the cluster substructures. The left node of the third plateau at $\sim 700 \mathrm{~km} \mathrm{~s}^{-1}$, shown by the open red circle, further splits the cluster core into additional substructures, as we illustrate below.

The caustic method uses the galaxies identified by the first threshold only to locate the celestial coordinates and the redshift of the cluster center. As discussed by Serra et al. (2011) and Serra \& Diaferio (2013), once the cluster is the dominant system in the field of view, the selection of the cluster center is not strongly affected by the selection of the first threshold of the binary tree. To identify the cluster members, the method first locates the caustics in the cluster redshift diagram, the plane of the line-of-sight velocity of the galaxies, and their projected separation from the cluster center (Figure 5). The caustics measure the galaxy escape velocity from the cluster, corrected by a function of the velocity anisotropy parameter 
Table 1

Redshift Catalog in the Fields of A2142

\begin{tabular}{|c|c|c|c|c|c|c|c|}
\hline ID & $\begin{array}{l}\text { SDSS ObjID } \\
\text { (DR13) }\end{array}$ & $\begin{array}{l}\text { R.A. } 2000 \\
\text { (deg) }\end{array}$ & $\begin{array}{l}\text { Decl.2000 }_{\text {(deg) }} \\
\text { (d) }\end{array}$ & $\begin{array}{c}m_{\mathrm{r}, \text { Petro, } 0} \\
\text { (mag) }\end{array}$ & $z$ & $u_{z}$ & $z$ source \\
\hline 26 & 1237662305125859528 & 239.041210 & 27.002581 & 16.950 & 0.06760 & 0.00003 & SDSS \\
\hline 27 & 1237662339475571013 & 239.041485 & 27.233463 & 17.867 & 0.18976 & 0.00007 & MMT \\
\hline 29 & 1237662339475571048 & 239.045342 & 27.174775 & 20.041 & 0.09611 & 0.00019 & Owers \\
\hline 30 & 1237662305662664876 & 239.046669 & 27.442754 & 18.429 & 0.17523 & 0.00007 & MMT \\
\hline
\end{tabular}

Note. (1) MMT: this study; (2) Owers: Owers et al. (2011); (3) SDSS: SDSS DR13; (4) Oegerle: Oegerle et al. (1995); The SDSS ObjIDs starting with "12" and "58" come from SDSS DR13 and DR7, respectively.

(This table is available in its entirety in machine-readable form.)

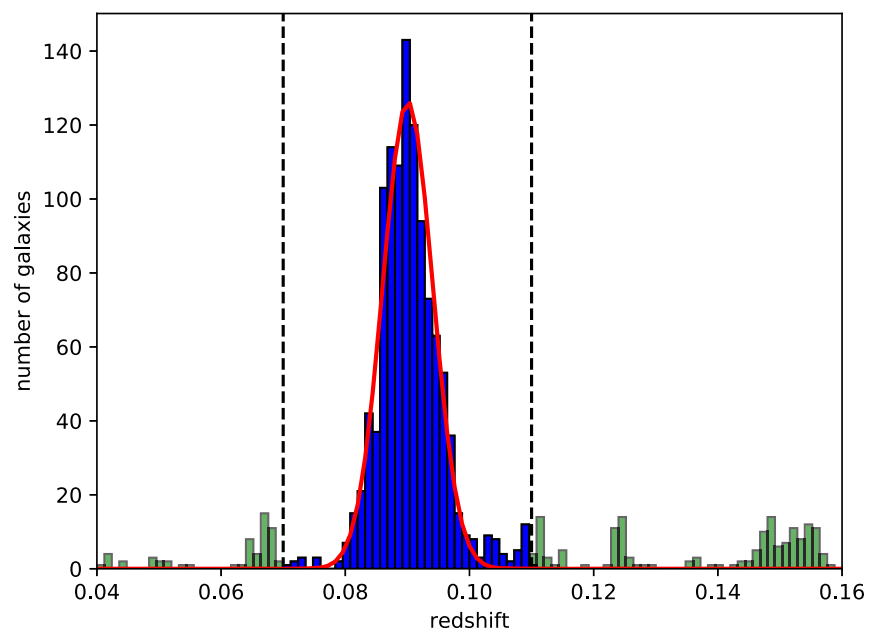

Figure 2. Redshift histogram of A2142. The green bars show the distribution of the field galaxies. The blue bars show the galaxies whose redshifts range from 0.07 to 0.11 (vertical dashed lines). The red solid line is the Gaussian fit result after the $3 \sigma$ clipping (Yahil \& Vidal 1977).

(see Serra et al. 2011 for details): the galaxies within the caustics are thus the members of the clusters. With this approach, we identify 868 galaxy members of A2142. This number is consistent with, albeit smaller than, the 1067 members identified with the $3 \sigma$ clipping method (see Section 2), and the 956 members identified by Owers et al. (2011) with the den Hartog and Katgert method (den Hartog \& Katgert 1996). The difference derives from the small fraction of interlopers misidentified as members by the caustic method: on average, only $2 \%$ of the caustic members within the cluster virial radius actually are interlopers and only $8 \%$ within three times the virial radius are interlopers (Serra \& Diaferio 2013).

Table 2 summarizes the basic properties of the substructures, from sub0 to sub19, identified by the second threshold at $\sim 870 \mathrm{~km} \mathrm{~s}^{-1}$, shown in Figure 4 . We also list the mean redshifts $z_{o}$ of the substructures with their uncertainty $\operatorname{err}_{z}=v_{\text {disp }} / \sqrt{N_{\text {gal }}}$, where $v_{\text {disp }}$ is the velocity dispersion and $N_{\mathrm{gal}}$ is the number of members of the substructure (Ivezic et al. 2014). Within the substructure sub0, the third threshold at $\sim 700 \mathrm{~km} \mathrm{~s}^{-1}$ identifies, at a lower level of the hierarchical clustering, six additional substructures that we list as sub00 to sub05 in Table 2. To assess whether these substructures correspond to physical systems rather than being chance alignments of unrelated galaxies, below we will combine these results with results from additional probes, including X-ray emission and gravitational lensing measurements.
Here, we also consider the relation between these substructures and the top five brightest (r-band Petrosian magnitudes) galaxies in the cluster (labeled BG1-5). They are all cluster members, except BG2. BG1 and BG2 are also well known brightest cluster galaxies. As shown in Figures 6 and 7, the brightest one-BG1-is in sub00, which is the core of the cluster. BG2 is a member of sub02 with a substantial velocity offset: its line-of-sight velocity is $312 \mathrm{~km} \mathrm{~s}^{-1}$ larger than the mean velocity of the members of sub02. The spiral galaxy BG3 is a member of sub17, a system in the outskirts of the cluster; unfortunately, this region is spectroscopically severely undersampled and we are unable to draw any solid conclusion. Finally, the elliptical galaxy BG4 and the interacting galaxy BG5 are members of sub04 and sub9, respectively. We will discuss the properties of sub9 and BG5 in Section 6.

\subsection{Results from the Dressler-Shectman Test}

The Dressler-Shectman (DS) test is largely used for the investigation of cluster substructures when optical spectroscopic data are available (Dressler \& Shectman 1988; Pinkney et al. 1996). The test requires the identification of the $N$ cluster members, which is usually obtained by removing the possible interlopers with the $3 \sigma$ clipping procedure (Yahil \& Vidal 1977). Around each cluster member $i$, we identify the $N_{\text {local }}=\sqrt{N}$ closest neighbors whose mean velocity $v_{\text {local }}^{i}$ and velocity dispersion $\sigma_{\text {local }}^{i}$ are compared with the mean velocity $v$ and velocity dispersion $\sigma$ of the $N$ members of the entire cluster. We thus define a local kinematic deviation for each cluster member

$$
\delta_{i}^{2}=\frac{N_{\text {local }}+1}{\sigma^{2}}\left[\left(v_{\text {local }}^{i}-v\right)^{2}+\left(\sigma_{\text {local }}^{i}-\sigma\right)^{2}\right] .
$$

The cumulative deviation $\Delta=\Sigma_{i} \delta_{i}$ is used as the test statistic to quantify the statistical significance of the presence of substructures. For a cluster with a Gaussian distribution of the member velocities, $\Delta$ is close to $N$. If the velocity distribution deviates from a Gaussian, $\Delta$ could vary significantly from $N$, either with or without substructures. Therefore, the statistical significance of the presence of substructures can be quantified by the ratio $p \equiv N\left(\Delta_{\text {simu }}>\Delta_{\text {obs }}\right) / N_{\text {simu }}$, where $\Delta_{\text {simu }}$ is the value of $\Delta$ estimated in $N_{\text {simu }}$ Monte Carlo simulations where the velocities of the galaxies are randomly shuffled, while the galaxy celestial coordinates are kept fixed, and $N\left(\Delta_{\text {simu }}>\Delta_{\text {obs }}\right)$ is the number of simulations where $\Delta_{\text {simu }}>$ $\Delta_{\text {obs }}$, where $\Delta_{\text {obs }}$ is the value obtained from the original data set. A small $p$ thus suggests a significant presence of substructures. 


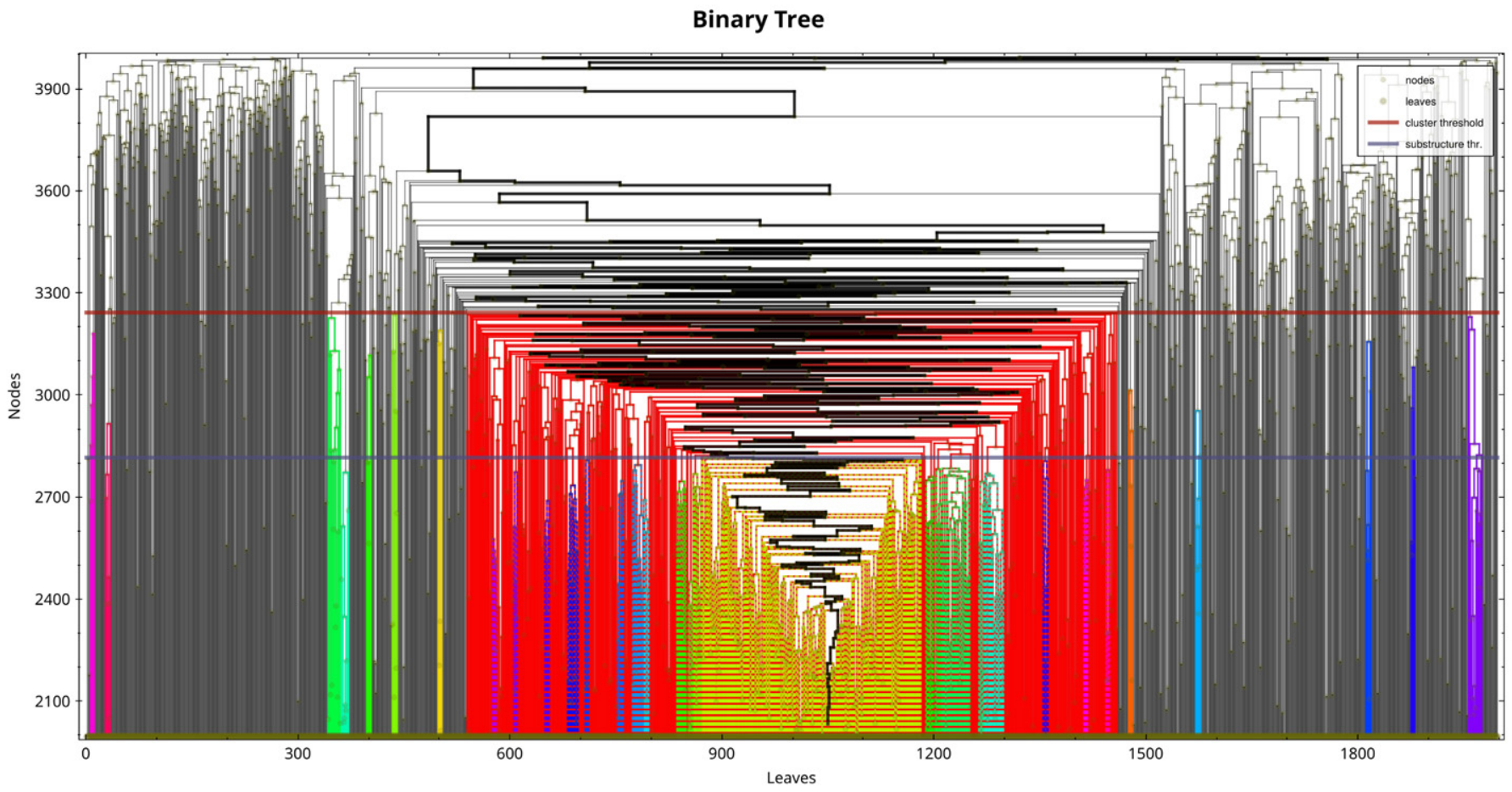

Figure 3. Dendrogram tree of 1997 redshifts in the range [0.01, 1.0]. The two solid horizontal lines are the first and second thresholds. The colors indicate different structures. The red part corresponds to sub0 in Table 2. The heavy black line shows the main branch of the binary tree.

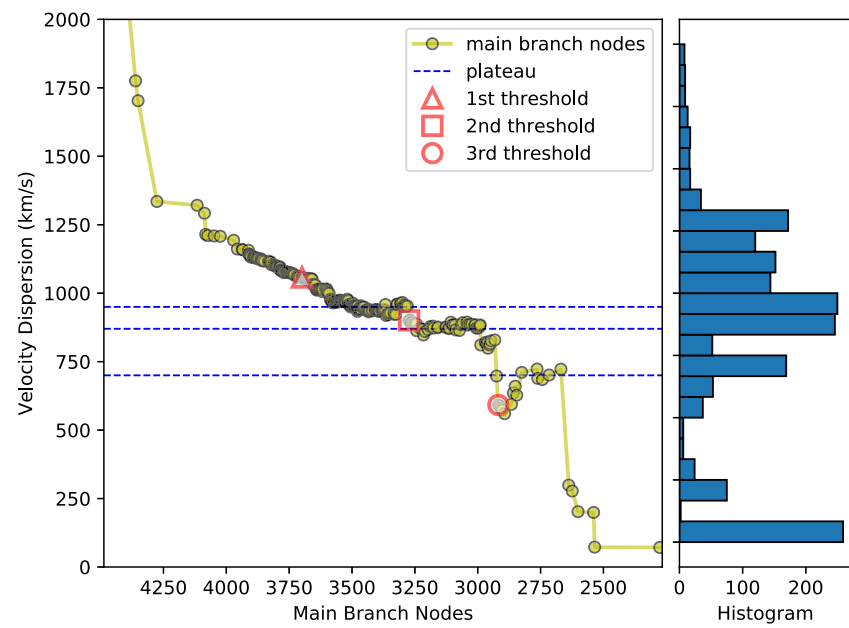

Figure 4. Velocity dispersion of the leaves of each node along the main branch of the binary tree of A2142. The histogram in the right panel shows the node numbers in different velocity dispersion bins. The blue dashed lines indicate the $\sigma$ plateaus. The red symbols are the selected thresholds.

We apply the DS test to our A2142 catalog. With $N=1067$ and $N_{\text {local }}=33$, we obtain $\Delta_{\text {obs }} / N=1.46$. We run $N_{\text {simu }}=$ 10,000 simulations and obtain $p=0.001$, which strongly indicates the existence of substructures. Figure 8 shows the $\delta_{i}$ of each cluster member on the plane of the sky. The radius of each circle is proportional to $e^{\delta_{i}}$. The blue and red circles represent galaxies with smaller and larger peculiar velocities with respect to the cluster mean velocity respectively. The clustering of circles with similar radii therefore suggests the presence of substructures. We stress that the large circles close to the edge of the field are unreliable, because the cluster outskirts are spectroscopically severely undersampled.

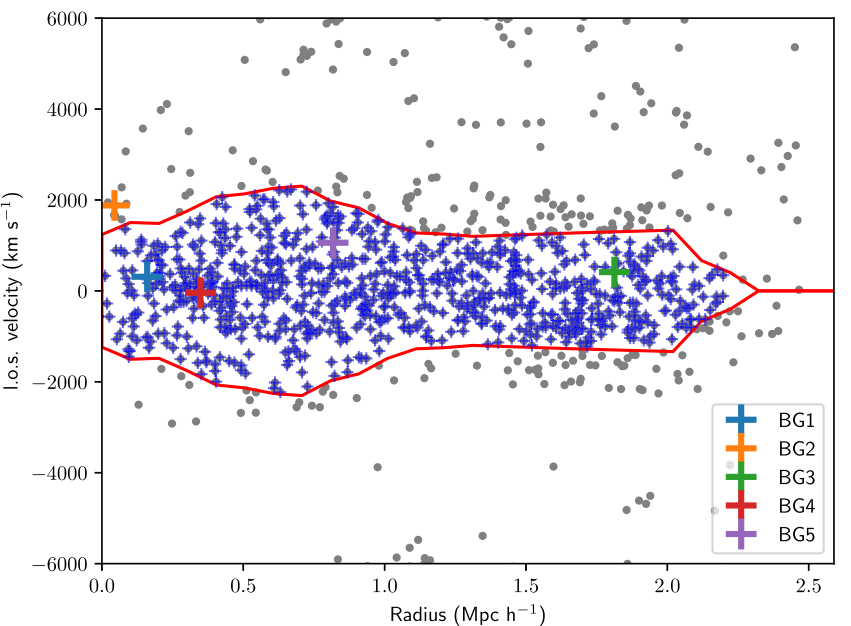

Figure 5. Redshift diagram of A2142. The solid lines show the caustic location. The blue crosses show the cluster members identified by the caustic method. We also show the location of the five brightest galaxies, BG1 -5 .

We now compare the results of the $\sigma$-plateau algorithm with the DS analysis. The areas including all the members of each substructure; sub1-19 and sub01-05 are denoted by ellipses in Figure 8. We label sub1, 2, 6, 7, 9, 11, 17, 18, and sub02; they overlap with clumps of circles derived from the DS analysis. Sub5, the purple structure in the NE, also overlaps a DS structure, but it is less secure because it is on the border of the field of view where the spectroscopic catalog is largely incomplete.

We note that the DS method searches for galaxy neighbors on the plane of the sky; therefore, clumps of galaxies that might be associated with real substructures might also contain cluster members that have velocities close to the cluster mean velocity. This event can commonly result in clumps of galaxies that are 
Table 2

Physical Properties of the Substructures

\begin{tabular}{lccc}
\hline \hline GroupID & $N_{\text {gal }}$ & $z_{o}$ & $v_{\text {disp }}\left(\mathrm{km} \mathrm{s}^{-1}\right)$ \\
\hline cluster & 868 & $0.08982 \pm 0.00010$ & $902 \pm 22$ \\
\hline sub0 & 311 & $0.08977 \pm 0.00017$ & $901 \pm 36$ \\
sub1 & 12 & $0.09590 \pm 0.00042$ & $431 \pm 91$ \\
sub2 & 7 & $0.08623 \pm 0.00011$ & $89 \pm 25$ \\
sub3 & 14 & $0.08776 \pm 0.00041$ & $462 \pm 90$ \\
sub4 & 12 & $0.08725 \pm 0.00041$ & $428 \pm 91$ \\
sub5 & 10 & $0.08903 \pm 0.00032$ & $307 \pm 72$ \\
sub6 & 40 & $0.08922 \pm 0.00032$ & $612 \pm 69$ \\
sub7 & 9 & $0.09645 \pm 0.00050$ & $447 \pm 111$ \\
sub8 & 27 & $0.08923 \pm 0.00033$ & $517 \pm 71$ \\
sub9 & 8 & $0.09459 \pm 0.00037$ & $310 \pm 83$ \\
sub10 & 16 & $0.09101 \pm 0.00050$ & $604 \pm 110$ \\
sub11 & 9 & $0.08976 \pm 0.00057$ & $513 \pm 128$ \\
sub12 & 6 & $0.09099 \pm 0.00046$ & $337 \pm 106$ \\
sub13 & 16 & $0.08672 \pm 0.00015$ & $180 \pm 32$ \\
sub14 & 8 & $0.09470 \pm 0.00030$ & $253 \pm 67$ \\
sub15 & 7 & $0.08921 \pm 0.00038$ & $300 \pm 86$ \\
sub16 & 6 & $0.08693 \pm 0.00014$ & $100 \pm 31$ \\
sub17 & 6 & $0.09235 \pm 0.00022$ & $160 \pm 50$ \\
sub18 & 6 & $0.08440 \pm 0.00019$ & $136 \pm 43$ \\
sub19 & 6 & $0.08614 \pm 0.00046$ & $335 \pm 106$ \\
\hline sub00 & 71 & $0.09041 \pm 0.00020$ & $591 \pm 43$ \\
sub01 & $0.08601 \pm 0.00031$ & $308 \pm 68$ \\
sub02 & $0.09547 \pm 0.00056$ & $344 \pm 108$ \\
sub03 & $0.08636 \pm 0.00094$ & \\
sub04 & $0.08911 \pm 0.00038$ & \pm 27 \\
sub05 & $0.08952 \pm 0.00047$ & \pm 216 \\
\hline & 6 & & \\
\hline
\end{tabular}

not plotted with the same color. A typical example is the DS clump at (R.A. $=239.460, \delta=27.33$ ) that overlaps sub1.

\section{Spatially Resolved ICM Redshift Measurements}

In this section, we investigate the dynamics of the ICM in the center of the cluster with spatially resolved X-ray redshift measurements. There are numerous observations of A2142 from XMM and Chandra. Since spatial resolution is crucial in this work, we focus on the Chandra data. In the Chandra data archive, we find seven observations available, as listed in Table 3. For X-ray redshift measurements, we only select the three most recent observations taken with ACIS-S to minimize possible systematic errors deriving from different calibrations. The total exposure time is $155.1 \mathrm{ks}$ after data processing. After examining the stacked image, we select a circle of $\sim 3$ arcmin for our spatially resolved spectral analysis. The total number of net counts in the $0.5-10 \mathrm{keV}$ energy band within this region is $\sim 10^{6}$.

Similar to Liu et al. (2016), we apply the contour binning technique (Sanders 2006) to separate the cluster field of view into independent regions based on the surface brightness contours. In order to acquire a more reliable redshift measurement in each region, we adopt a threshold signal-tonoise ratio $(\mathrm{S} / \mathrm{N})$ larger than that in Liu et al. (2016). We separate the circle of 3 arcmin around the cluster center into 26 regions with $\mathrm{S} / \mathrm{Ns}$ larger than 200 . Figure 9 shows the map of these regions.

The spectra are fitted with Xspec v12.9.1 (Arnaud et al. 1996) in the $0.5-10 \mathrm{keV}$ band. Because the chip S3 is entirely covered by the cluster emission, we extract the background from the chip S1. We also check the background
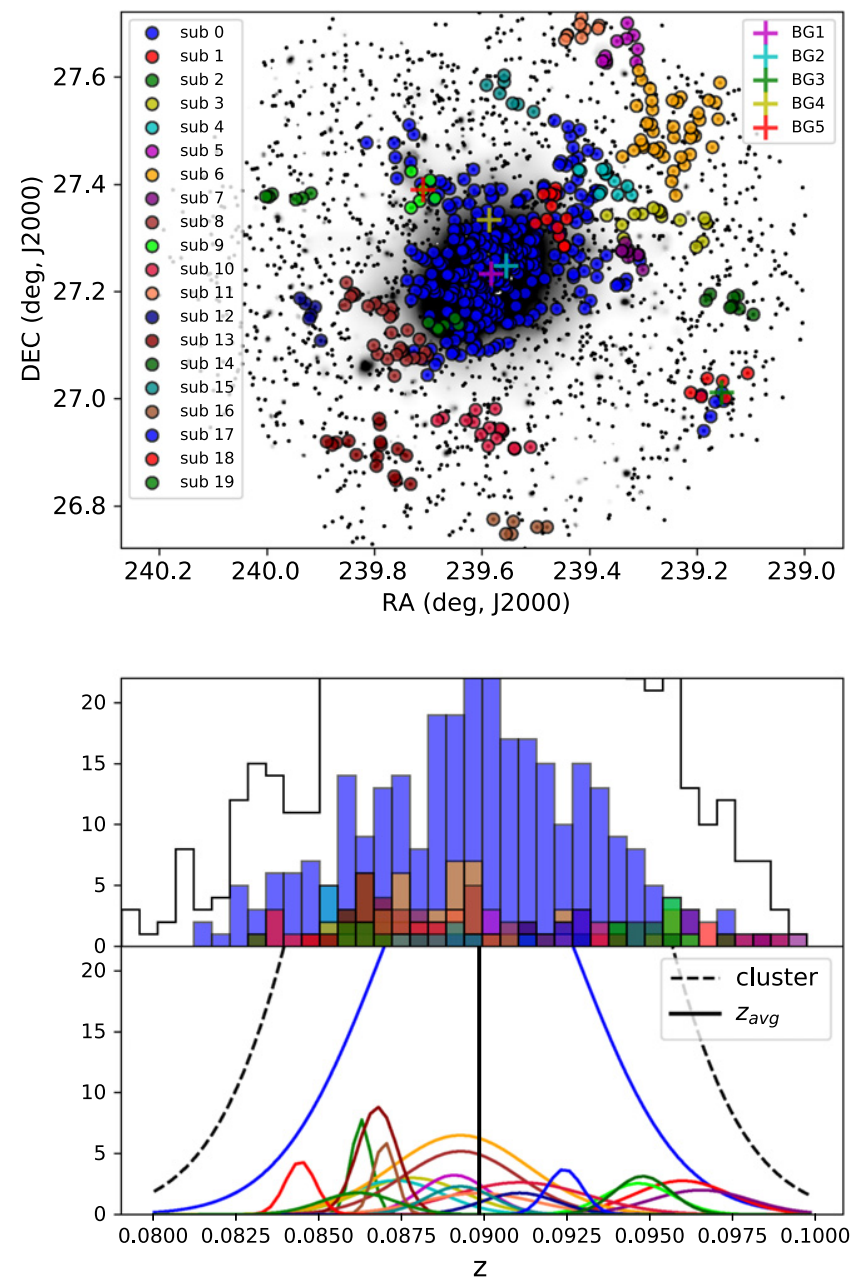

Figure 6. The upper panel shows the distribution on the sky of the A2142 substructures identified with the second threshold. The white contours and grayscale of the background image show the X-ray surface brightness from the combined X-ray observations of XMM (Tchernin et al. 2016). The middle and bottom panels show the velocity histograms of the substructures and their best Gaussian fits. The black vertical line shows the position of the mean redshift $z_{\text {avg }}=0.08982 \pm 0.00010$ as a reference.

modeled from the "blank sky" data set, and find that the two results are consistent. To model the X-ray emission from a projected region, we use the double-apec thermal plasma emission model (Smith et al. 2001). The double-temperature thermal model is helpful to reduce the possible bias in the measurement of the iron line centroid due to the presence of unnoticed thermal components along the line of sight (Liu et al. 2015). Galactic absorption is described by the model tbabs (Wilms et al. 2000). The ICM temperature, the metallicity, the redshift, and the normalization are all set as unconstrained at the same time. The redshifts of the two components in the model are always linked. Considering the large parameter space to explore, for the fitting we adopt a Monte Carlo Markov Chain (MCMC) method. The chain is generated by the Goodman-Weare algorithm (Jonathan Goodman 2010), with 10 walkers, $10^{4}$ burn-in steps, and a total length of $10^{6}$ steps. After the fitting, chains are top-hat-filtered according to the following ranges: temperature from $0.1 \mathrm{keV}$ to $25 \mathrm{keV}$, metallicity from 0.001 to 2 , and redshift from 0.05 to 0.15 . The best-fit parameters and their uncertainties are estimated from these filtered chains. The best-fit results of the regions are listed in Table 4. 

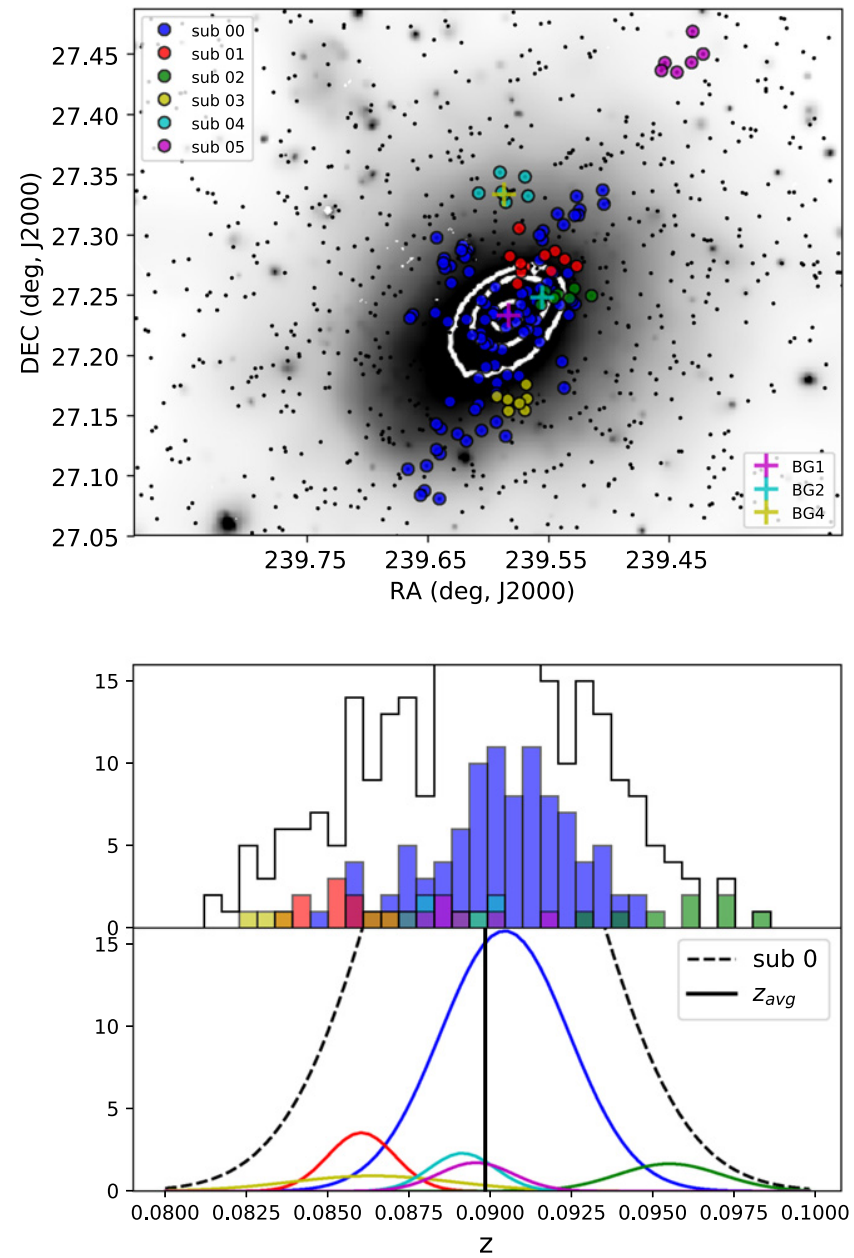

Figure 7. Same as Figure 6, but for the A2142 substructures identified with the third threshold.

We present our results in the form of a redshift map, shown in Figure 10. Region 7 has a redshift uncertainty that is larger than 0.01 and appears white. The most prominent feature emerging from the redshift map is that seven regions form an elliptical annulus, marked with the two black ellipses in Figure 10, with a mean redshift larger than its surrounding regions. Specifically, its redshift is $0.0916 \pm 0.0011$. The velocity difference between the annulus and the cluster average is therefore $810 \pm 330 \mathrm{~km} \mathrm{~s}^{-1}$. The emergence of this highredshift annulus appears to be consistent with the scenario where the ICM is sloshing due to one or more perturbations. However, the limited spatial resolution of the map implies that we can only derive rough estimates of both the size and the redshift of the annulus.

Additionally, we note that the cluster core is surrounded by another high-redshift annulus with redshift $0.0911 \pm 0.0010$. The velocity difference between this annulus and the cluster average is $660 \pm 300 \mathrm{~km} \mathrm{~s}^{-1}$. A possible interpretation of this feature is that is a small-scale sloshing that generates a "wave"like motion of the ICM. Alternatively, this feature could be the signature of the rotation of the cool core, which is also an event suggested by the spiral-like structures observed in other clusters (Laganá et al. 2010). Clearly, the projection effects and the large systematic uncertainties in the X-ray redshift measurements require deeper observations with the

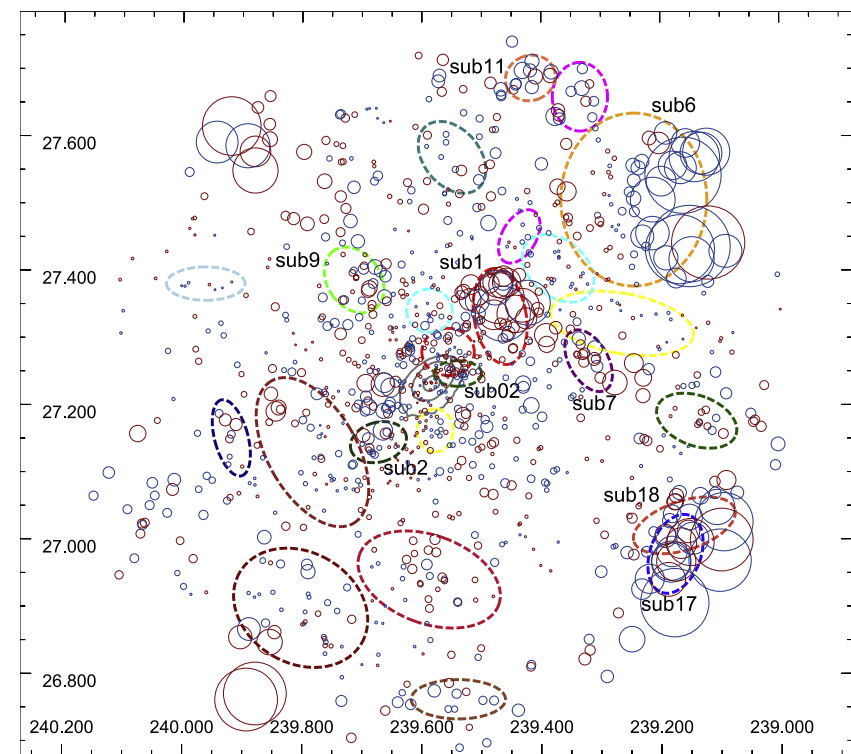

Figure 8. Results of the DS analysis based on the 1067 member galaxies identified by the $3 \sigma$ clipping procedure on galaxy velocities. The blue and red circles represent galaxies with velocities smaller and larger than the cluster mean velocity, respectively. The radius of each circle is proportional to $e^{\delta_{i}}$. The ellipses mark the substructures identified with the $\sigma$-plateau algorithm. The colors of these substructures are the same as in Figures 6 and 7. We only label the names of the substructure ellipses that overlap with a clump of DS circles. The gray contour levels in the center show the distribution of the X-ray emission.

Table 3

List of XMM and Chandra Observations of A2142

\begin{tabular}{lccr}
\hline \hline CCD & ObsID & Exptime $(\mathrm{ks})$ & \multicolumn{1}{c}{ Date } \\
\hline EPIC & 0111870101 & 35.5 & 2002 Jul 20 \\
EPIC & 0111870401 & 13.7 & 2002 Sep 08 \\
EPIC & 0674560201 & 59.4 & 2011 Jul 13 \\
ACIS-S & 1196 & 11.4 & 1999 Sep 04 \\
ACIS-S & 1228 & 12.1 & 1999 Sep 04 \\
ACIS-I & 5005 & 44.5 & 2005 Apr 13 \\
ACIS-I & 7692 & 5.0 & 2007 May 07 \\
ACIS-S & $\mathbf{1 5 1 8 6}$ & $\mathbf{8 9 . 9}$ & 2014 Jan 19 \\
ACIS-S & $\mathbf{1 6 5 6 4}$ & $\mathbf{4 4 . 5}$ & 2014 Jan 22 \\
ACIS-S & $\mathbf{1 6 5 6 5}$ & $\mathbf{2 0 . 8}$ & 2014 Jan 24 \\
\hline
\end{tabular}

Note. The three most recent observations we used for the X-ray redshift measurements are bold.

next-generation X-ray bolometers to pin down the appropriate scenario.

\section{Weak-lensing Data and Analysis}

A2142 is among the seven nearby merging clusters targeted by the Subaru weak-lensing analysis of Okabe \& Umetsu (2008), who performed a detailed comparison of the weaklensing mass distribution with the $\mathrm{X}$-ray brightness and cluster galaxy distributions. Umetsu et al. (2009) conducted a combined weak-lensing and Sunyaev-Zel'dovich effect analysis of A2142, along with three other X-ray luminous clusters targeted by the 7-element AMiBA project (Ho et al. 2009) to determine the hot gas fractions in the clusters in combination with X-ray temperatures. The Umetsu et al. (2009) weaklensing analysis of A2142 is based on the same Subaru images as the analysis in Okabe \& Umetsu (2008), but their improved 


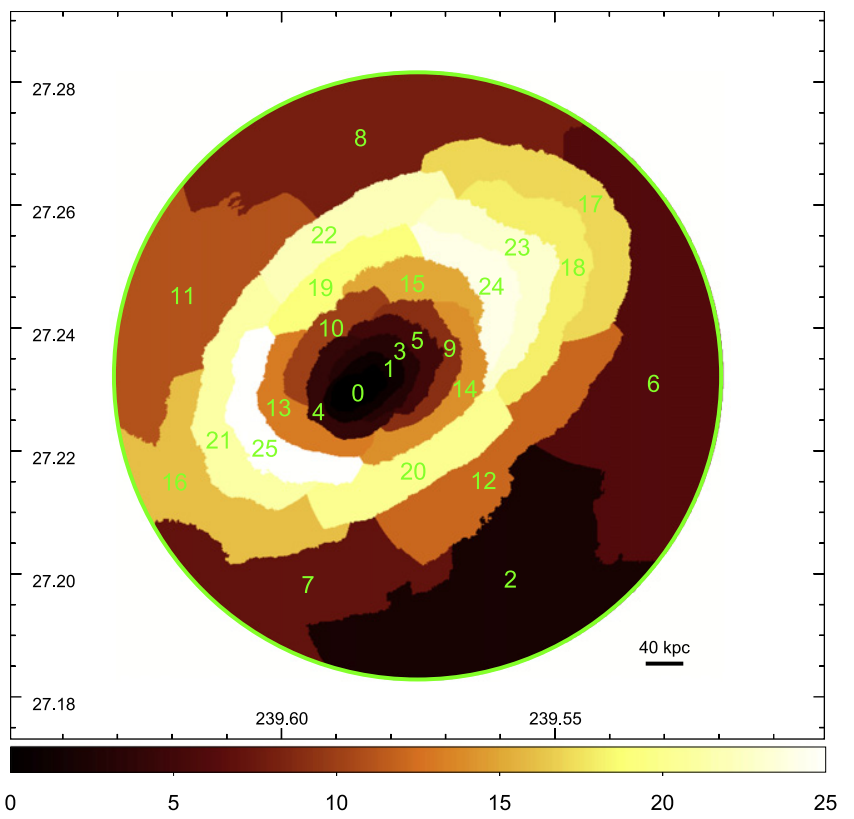

Figure 9. Region map produced on the basis of the surface brightness contours. The color bar denotes the serial number of the regions and has no physical meaning.

Table 4

X-Ray Fitting Results

\begin{tabular}{lccc}
\hline \hline Region ID & X-Ray redshift & Region ID & X-Ray redshift \\
\hline 00 & $0.0834_{-0.0007}^{+0.0007}$ & 13 & $0.0906_{-0.0031}^{+0.0051}$ \\
01 & $0.0911_{-0.0017}^{+0.0015}$ & 14 & $0.0862_{-0.0049}^{+0.0022}$ \\
02 & $0.0862_{-0.0082}^{+0.0075}$ & 15 & $0.0849_{-0.0004}^{+0.0015}$ \\
03 & $0.0911_{-0.0013}^{+0.0011}$ & 16 & $0.0972_{-0.0085}^{+0.0020}$ \\
04 & $0.0872_{-0.0001}^{+0.0025}$ & 17 & $0.0892_{-0.0007}^{+0.0032}$ \\
05 & $0.0866_{-0.0025}^{+0.0019}$ & 18 & $0.0954_{-0.0023}^{+0.0040}$ \\
06 & $0.0877_{-0.0054}^{+0.0055}$ & 19 & $0.0845_{-0.0022}^{+0.0008}$ \\
07 & $0.0898_{-0.0194}^{+0.0184}$ & 20 & $0.0920_{-0.0034}^{+0.0016}$ \\
08 & $0.0876_{-0.0054}^{+0.0073}$ & 21 & $0.0852_{-0.0016}^{+0.0032}$ \\
09 & $0.0879_{-0.0013}^{+0.0015}$ & 22 & $0.0913_{-0.0017}^{+0.0016}$ \\
10 & $0.0862_{-0.0013}^{+0.0016}$ & 23 & $0.0897_{-0.0033}^{+0.0035}$ \\
11 & $0.0879_{-0.0041}^{+0.0055}$ & 24 & $0.0876_{-0.0015}^{+0.0011}$ \\
12 & $0.0873_{-0.0020}^{+0.0011}$ & 25 & $0.0931_{-0.0029}^{+0.0030}$ \\
\hline
\end{tabular}

method of selecting blue+red background galaxies in $g^{\prime} R_{\mathrm{C}}$ color-magnitude space increased the size of the background sample by a factor of 4 relative to that of Okabe \& Umetsu (2008). With the improved background selection, Umetsu et al. (2009) obtained a virial mass estimate of $M_{\mathrm{vir}}=$ $15.2_{-2.3}^{+3.1} \times 10^{14} M_{\odot}$ and a concentration of $c_{\text {vir }}=5.5 \pm 1.1$ (see their Table 4) from tangential shear fitting assuming a spherical Navarro-Frenk-White halo (Navarro et al. 1996, NFW hereafter).

Here, we revisit the weak-lensing properties of A2142 by performing a weak-lensing analysis using our most recent shape measurement pipelines employed by the CLASH collaboration (Umetsu et al. 2014) and the LoCuSS collaboration (Okabe \& Smith 2016). We analyze the Subaru/Suprime-Cam $g^{\prime} R_{\mathrm{C}}$ images reduced by Okabe \& Umetsu (2008), and apply the same color-magnitude cuts as presented in Umetsu et al. (2009)

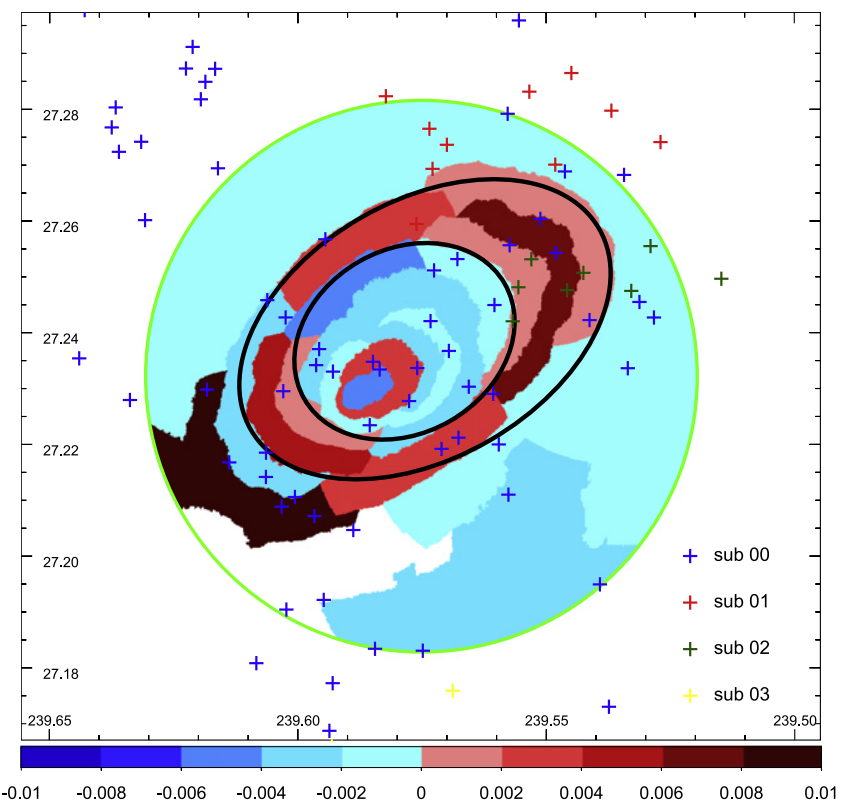

Figure 10. Redshift map of A2142. The color bar indicates the redshift difference with respect to the average X-ray redshift $z_{X}=0.0889 \pm 0.0009$. The black ellipses roughly mark the elliptical annulus with redshift larger than the surroundings.

to select background galaxies. We use the Subaru $R_{\mathrm{C}}$-band images for the shape measurement, as done in previous work. Briefly summarizing, the key common feature in our shape measurement pipelines is that only those galaxies detected with sufficiently high significance are used to model the isotropic point-spread-function correction as a function of object size and magnitude (for details, see Umetsu et al. 2014; Okabe \& Smith 2016). For each galaxy, we apply a shear calibration factor, $g \rightarrow g / 0.95$, to account for the residual correction estimated using simulated Subaru/Suprime-Cam images (Umetsu et al. 2014; Okabe \& Smith 2016). All galaxies with usable shape measurements are then matched with those in the blue+red background samples. Our conservative selection criteria yield a mean surface number density of $n_{\mathrm{g}} \simeq 25$ galaxies $\operatorname{arcmin}^{-2}$ for the weak-lensing-matched background catalog, compared to $n_{\mathrm{g}} \simeq 30$ galaxies $\operatorname{arcmin}^{-2}$ found by Umetsu et al. (2009). We checked that our results from the two different pipelines (Umetsu et al. 2014; Okabe \& Smith 2016) are robust and entirely consistent with each other.

In Figure 11 we show the tangential reduced shear profile in units of projected mass density, $\Delta \Sigma_{+}(R)=\Sigma_{\mathrm{c}} g_{+}(R)$, with $\Sigma_{\mathrm{c}} \simeq 5.5 \times 10^{15} M_{\odot} \mathrm{Mpc}^{-2}$ as the critical surface mass density for lensing and $g_{+}(R)$ as the azimuthally averaged reduced tangential shear as a function of cluster-centric radius $R$. We fit the $\Delta \Sigma_{+}(R)$ profile with a spherical NFW halo using log-uniform priors for $M_{200 c}$ and $c_{200 c}$ in the range $0.1<$ $M_{200 \mathrm{c}} /\left(10^{15} M_{\odot} h^{-1}\right)<10$ and $0.1<c_{200 \mathrm{c}}<10$, where $h \equiv H_{0} /\left(100 \mathrm{~km} \mathrm{~s}^{-1} \mathrm{Mpc}^{-1}\right)=0.704$. The error analysis includes the contribution from cosmic noise due to the uncorrelated large-scale structure projected along the line of sight (Hoekstra 2003), as well as galaxy shape noise and measurement errors. The mass and concentration parameters are constrained as $M_{200 \mathrm{c}}=(13.0 \pm 2.7) \times 10^{14} M_{\odot}$ and $c_{200 \mathrm{c}}=$ $4.1 \pm 0.8$, or $\quad M_{\mathrm{vir}}=(16.1 \pm 3.7) \times 10^{14} M_{\odot}$ and $c_{\mathrm{vir}}=$ $5.5 \pm 1.1$, in good agreement with the results of Umetsu et al. (2009). The uncertainties are slightly larger than those 


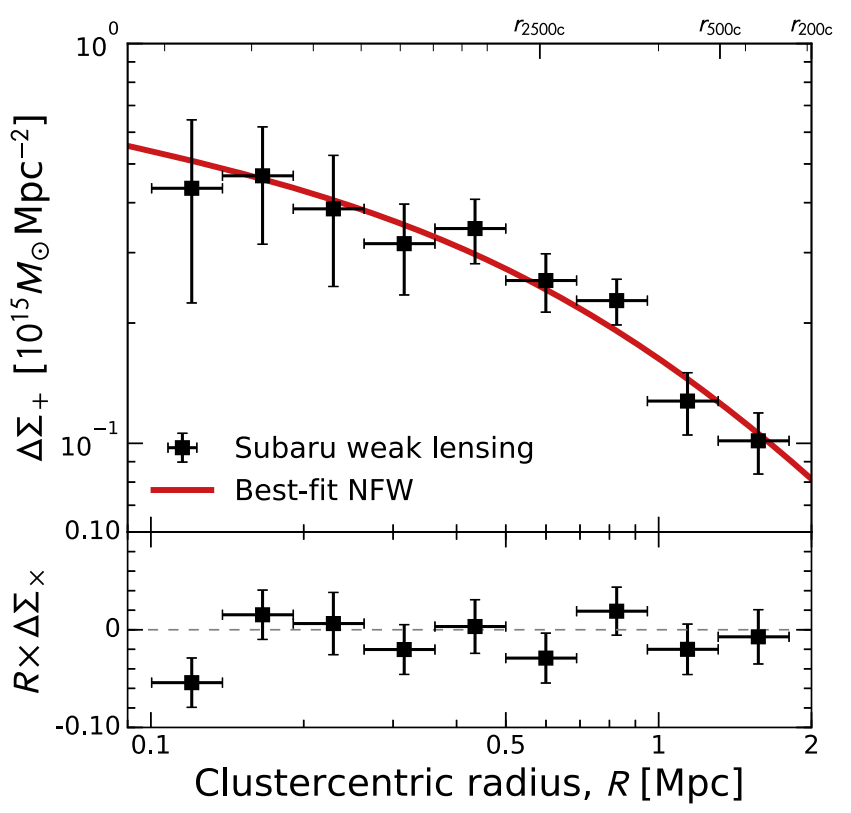

Figure 11. Tangential reduced shear profile (upper panel, black squares) of A2142 derived from our Subaru/Suprime-Cam weak-lensing observations, shown in units of projected mass density. The thick solid (red) line corresponds to the best-fit NFW profile. The lower panel shows the $45^{\circ}$ rotated $\times$ component, which is consistent with a null signal within $2 \sigma$ at all cluster radii.

estimated by Umetsu et al. (2009), who did not account for the cosmic noise contribution.

The mass estimated with the weak-lensing analysis is consistent with the mass profile estimated with the caustic method, based on the amplitude of the caustics in the redshift diagram (Figure 5). The caustic method estimates the radius $r_{200}=2.15 \mathrm{Mpc}$ and the mass within $r_{200}, M_{200}=(11.5 \pm 3.7) \times 10^{14} M_{\odot}$. This agreement confirms the results of Geller et al. (2013), who show that, for a sample of 19 clusters, the caustic and weak-lensing masses within $r_{200}$ agree to within $\sim 30 \%$, similar to the early results of Diaferio et al. (2005).

In Figure 12, we show the weak-lensing mass map of A2142. The mass map is smoothed with a Gaussian of FWHM $=1 ! 5$. The mass map exhibits an extended structure elongated along the northwest-southeast direction, consistent with the direction of elongation of the X-ray emission. We compare the mass map with the previous map reconstructed from the old shape catalog (Okabe \& Umetsu 2008). Since the number density of background galaxies for this analysis is slightly lower than that of the previous analysis, the Gaussian FWHM used for the map is $1 ! 5$, larger than that for the old map (1!0). Okabe \& Umetsu (2008) found the main peak at $\sim 11 \sigma$ and a possible substructure at $\sim 3 \sigma$ in the northwest region. The noise level was computed with the theoretical estimations from the number density and the variance of ellipticity. Here, we find the main peak at $\sim 10 \sigma$ and the northwest substructure at $\sim 3.3 \sigma$. The noise is computed by the bootstrap re-sampling with 3000 realizations using random ellipticity catalog in order to conservatively evaluate a spatially dependent noise level caused by sparse galaxy distributions. Although the techniques are revised from the previous study, the overall mass distributions are similar to each other. In this paper, we compare the new map with the distributions of the substructures determined by the dynamical method.

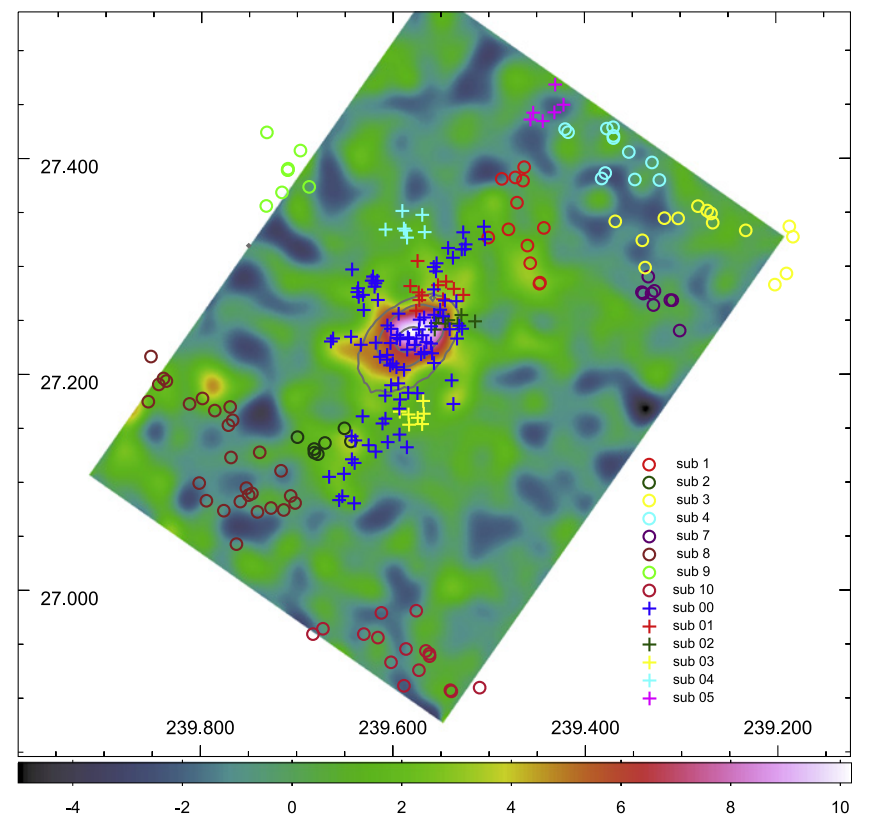

Figure 12. Weak-lensing mass map superimposed with the substructures identified with the $\sigma$-plateau algorithm. The color code shows the weak-lensing $\mathrm{S} / \mathrm{N}$, namely the surface mass density in units of the $1 \sigma$ reconstruction error.

\section{Combined Analysis and Discussion}

In this section, we combine the results of the above analyses to attempt to construct a portrait of the internal structure and dynamics of A2142. We also compare our results with the previous results by Owers et al. (2011) and Einasto et al. (2018), who used different methods to detect substructures in A2142.

We first compare the optical substructures identified with the $\sigma$-plateau algorithm with the ICM redshift map, as shown in Figure 10. The ICM redshift map only covers the region within 3 arcmin from the cluster center, so Figure 10 only shows the substructures sub00 to sub03 identified with the third threshold trimming the binary tree of A2142. Figure 10 does not show any clear correlation between the redshift and spatial distributions of the optical substructures and the ICM distribution and redshift. This result suggests that the dynamics of the ICM in this region have decoupled from the dynamics of the galaxies. This behavior is not unexpected in merging systems, because galaxies approximately behave like collisionless components, unlike the ICM.

In Figure 12, we superimpose the substructures identified by the $\sigma$-plateau algorithm on the weak-lensing mass map. Figure 12 shows that the shape of the main halo of the mass map and the distribution of the member galaxies of the cluster core sub00 are consistent with each other. Moreover, the $\sim 3.3 \sigma$ excess in the weak-lensing signal, located $\sim 7^{\prime}-8^{\prime}$ northwest of the cluster center, coincides with sub1. This match supports the results of Okabe \& Umetsu (2008), who found that this northwest mass substructure is associated with a slight excess of galaxies in the color-magnitude relation of the A2142 galaxies, lying $\sim 5^{\prime}$ ahead of the northwest edge of the central X-ray core: we confirm that 6 out of the 12 members of sub1 do indeed belong to this group of galaxies identified by Okabe $\&$ Umetsu (2008). Finally, we also find marginally significant excesses in the weak-lensing map that coincide with sub04, sub01, and sub2. These results demonstrate that our $\sigma$-plateau substructure identification algorithm can efficiently detect 


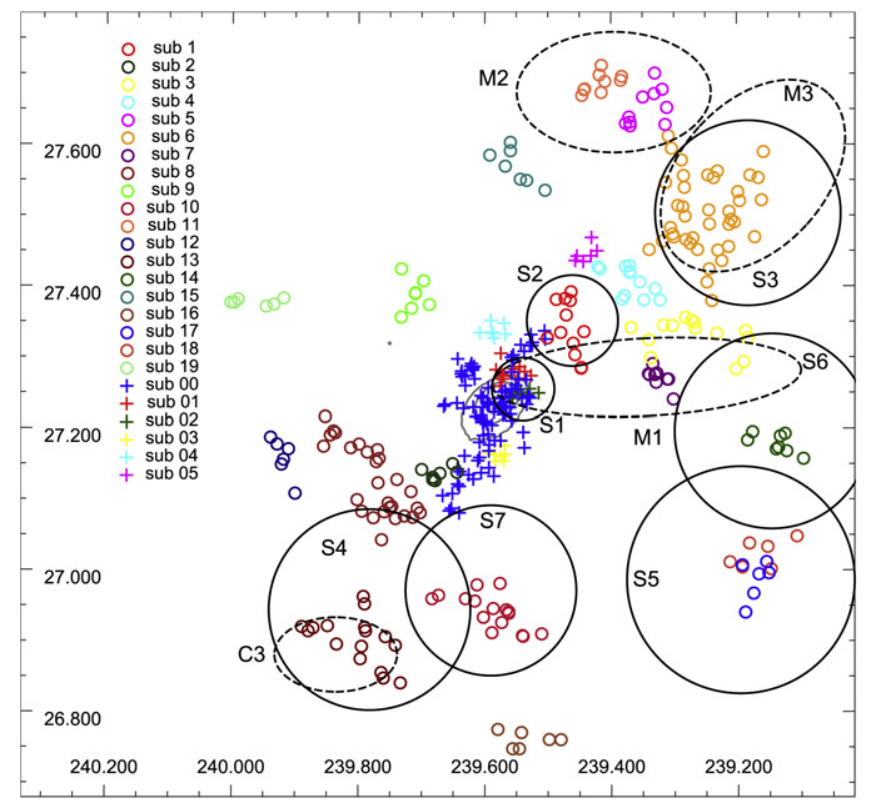

Figure 13. Substructures identified with the $\sigma$-plateau algorithm and with other methods in the literature. S1-S7, shown as black circles, represent the locations and sizes of the substructures identified with the $\kappa$-test by Owers et al. (2011). The dashed ellipses show the approximate locations and sizes of the structures identified by Einasto et al. (2018).

structures that are around the detection limit of the weaklensing signal.

Figure 13 compares the substructures identified with the $\sigma$-plateau algorithm with the substructures identified in previous work. Owers et al. (2011) detected seven substructures, S1-S7, from the projected galaxy surface density distribution and a $\kappa$-test on the local kinematics of the galaxies, where the $\kappa$-test identifies kinematic substructures by comparing the local velocity distribution to the global velocity distribution (Colless \& Dunn 1996). The open circles in Figure 13 show the location and size of the substructures identified by Owers et al. (2011), according to their Figure 12.

Einasto et al. (2018) identified four substructures, M1-M3 and C3, by analyzing the position and velocities of member galaxies with the mclust package, which is based on the analysis of a finite mixture of distributions, in which each mixture component corresponds to a different subgroup. To provide a qualitative impression of the locations and sizes of these structures we plot four ellipses in Figure 13, according to the information that can be inferred by eye from Figures 4 and 6 of Einasto et al. (2018).

We can see that sub1 overlaps with S2 of Owers et al. (2011), which is consistent with the most obvious DS substructure (see Figure 8). Sub5 and sub11 coincide with substructure M2 in Einasto et al. (2018). Our most prominent substructure on the northwest, sub6, coincides with S3 and M3; sub6 is also clearly detected by the DS analysis (Figure 8). Sub7 lies within M1; we note that the rest of the galaxies associated with M1 by Einasto et al. (2018) are identified as substructure members by neither Owers et al. (2011) nor our $\sigma$-plateau algorithm. Sub10 overlaps with S7. Sub13 overlaps with S4 and the component C3 of Einasto et al. (2018) (see their Figure 4). Sub17 and sub18 overlap with S5 and also appear as clumps of the DS analysis.

All the remaining substructures, except sub8, that are identified by the $\sigma$-plateau algorithm and do not have a

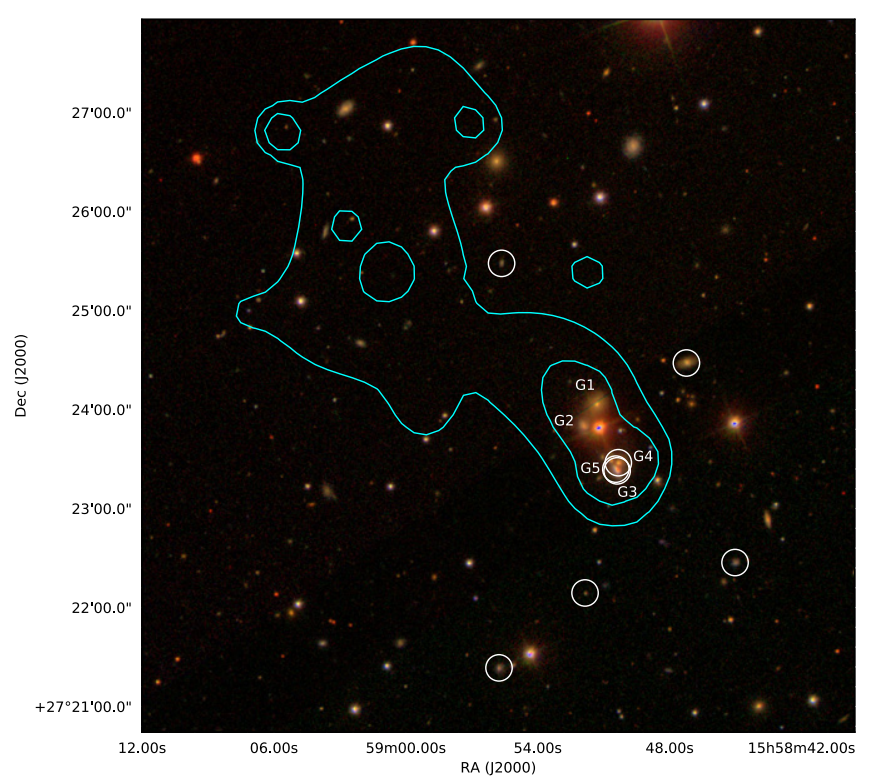

Figure 14. Zoomed-in image of the substructure sub9. The SDSS RGB image converted from the $i, r, g$ bands is superimposed with the X-ray contours of the gas; the white circles are the members of sub9 according to the $\sigma$-plateau algorithm. The five galaxies in the image are labeled G1-G5, as in Eckert et al. (2017). G3 and G4 are confirmed to be members of sub9.

correspondence with substructures from previous analyses, have 14 members at most, indicating that either the catalogs used in previous analysis did not contain enough galaxies or these structures, if they are not chance alignment of unrelated galaxies, are too poor to be reliably identified by other methods. We conclude that, overall, this comparison shows a remarkable agreement between the different substructure identifications.

As shown in Section 4, sub9 in the northeast of the cluster is identified by both the DS method and the $\sigma$-plateau algorithm. The circles associated with the galaxies according to the DS method in that region of the sky (Figure 8) have both red and blue colors: most of the red circles are members of sub9, whereas the blue circles, which have redshifts smaller than the sub9 redshift, are not members of sub9. The X-ray images from both XMM-Newton (Eckert et al. 2014) and Chandra (Eckert et al. 2017) show the presence of a faint gas component associated with this structure. For this X-ray emitting gas, Eckert et al. (2014, 2017) determine an average temperature of $\sim 1.4 \mathrm{keV}$, appropriate for a system with a total mass of $\sim 5.1 \times 10^{13} M_{\odot}$ (Vikhlinin et al. 2006). According to the analysis of Munari et al. (2013, 2014), this mass is consistent with the mass suggested by the velocity dispersion of $310 \mathrm{~km} \mathrm{~s}^{-1}$ that we measure for the members of the optical substructure. Figure 14 shows an optical image of sub9, with the open white circles indicating its members according to the $\sigma$-plateau algorithm. We also indicate the five galaxies, G1-G5, associated by Eckert et al. $(2014,2017)$ with the clump of hot gas. Our analysis confirms that G3, which we name BG5 in Section 3.1, and G4 and G5 are members of sub9. On the contrary, G1 and G2 are not members: in fact, G1 and G2 have a velocity $1700 \mathrm{~km} \mathrm{~s}^{-1}$ smaller and $1130 \mathrm{~km} \mathrm{~s}^{-1}$ larger, respectively, than the mean redshift of sub9. The redshift of sub9 $-z=0.09459-$ is significantly higher than the average redshift of the cluster- $z=0.08982$ - and shows that this system is falling into the cluster at a greater speed. 
We conclude that the optical substructure sub9 and the faint $\mathrm{X}$-ray clump originate from the same group that is currently falling into the cluster: the ram pressure of the ICM acting on the group gas, but not on its galaxy members, is a plausible explanation for the displacement on the sky between the galaxies and the X-ray emission.

\section{Conclusions}

We investigate the dynamics of A2142 by comparing the properties of the substructures in the galaxy distribution, the line-of-sight velocity field of the ICM derived from the spatially resolved X-ray spectroscopy, and the weak-lensing mass distribution. Our main results are as follows:

1. Based on a new and extended catalog of spectroscopic redshifts within $\sim 3.5 \mathrm{Mpc}$ from the cluster center, we identify a number of substructures with the $\sigma$-plateau algorithm. The distribution of the substructures appears consistent with results obtained with other methods, including the DS method, the $\kappa$-test, and the mclust algorithm.

2. Most substructures have a number of galaxy members $\sim 10$ (see Table 2), indicating that there is no sign of recent or ongoing major mergers and suggesting a scenario where the cold fronts observed in A2142 in $\mathrm{X}$-rays originate from core-sloshing induced by minor mergers.

3. In the northeast outskirts, the galaxy substructure sub9 matches a falling gas clump observed in X-rays; the slight displacement between the positions of the galaxies and the gas might be due to ram pressure on the hot gas.

4. The shape of the central substructure sub00 is consistent with the projected weak-lensing mass map. Several substructures also coincide with the weak-lensing mass excesses.

5. With spatially resolved X-ray spectroscopy based on Chandra data, we measure the line-of-sight velocity distribution of the ICM within $\sim 0.35 \mathrm{Mpc}$ from the cluster center. We find an annulus near the X-ray cold fronts with a redshift significantly larger than the surroundings, corresponding to a velocity $810 \pm 330 \mathrm{~km} \mathrm{~s}^{-1}$ larger than the cluster mean velocity. We also find that the core is surrounded by high-redshift gas, with a velocity $660 \pm$ $300 \mathrm{~km} \mathrm{~s}^{-1}$ larger than the cluster redshift. The features we observe in the X-ray redshift map appear to be consistent with the core-sloshing scenario suggested in previous work.

Deeper photometric and spectroscopic observations of the field can clearly provide more detailed and solid results. The spatially resolved X-ray redshift measurements will improve with advanced future X-ray bolometers. In particular, with the X-ray IFU on board, the Advanced Telescope for High-ENergy Astrophysics (Athena) will remarkably extend the application of this method. Moreover, a more precise weak-lensing measurement of the projected mass distribution will be of great help to confirm the relation between the total mass distribution and the galaxy substructures we find here.

We thank the anonymous referee whose comments substantially improved the presentation of our results. We thank Dominique Eckert, Sabrina de Grandi, and Maria Chiara Rossetti for the results of XMM-Newton and their helpful comments. We sincerely thank Margaret Geller for her insightful suggestions. This work was supported by the National Natural Science Foundation of China under grants No. 11403002, the Fundamental Research Funds for the Central Universities and Scientific Research Foundation of Beijing Normal University. A.D. and H.Y. acknowledge partial support from the grant of the Italian Ministry of Education, University and Research (MIUR) (L. 232/2016) "ECCELLENZA1822_D206 - Dipartimento di Eccellenza 2018-2022 Fisica" awarded to the Department of Physics of the University of Torino. A.D. also acknowledges partial support from the INFN Grant InDark. P.T. is supported by the Recruitment Program of High-end Foreign Experts and he gratefully acknowledges hospitality of Beijing Normal University. K.U. acknowledges support from the Academia Sinica Investigator Award and from the Ministry of Science and Technology of Taiwan (grant MOST 103-2628-M-001-003-MY3).

\section{ORCID iDs}

Ang Liu (刘昂) (10 https://orcid.org/0000-0003-3501-0359

Heng Yu (余恒) (10 https://orcid.org/0000-0001-8051-1465

Paolo Tozzi (ib https://orcid.org/0000-0003-3096-9966

Ho Seong Hwang (i) https://orcid.org/0000-0003-3428-7612

Keiichi Umetsu (10) https://orcid.org/0000-0002-7196-4822

Nobuhiro Okabe (iD https://orcid.org/0000-0003-2898-0728

Li-Lan Yang (杨里岗) (10 https://orcid.org/0000-0002-8434-880X

\section{References}

Albareti, F. D., Allende Prieto, C., Almeida, A., et al. 2017, ApJS, 233, 25 Arnaud, K. A. 1996, in ASP Conf. Ser. 101, Astronomical Data Analysis Software and Systems V, ed. G. H. Jacoby \& J. Barnes (San Francisco, CA: ASP), 17

Caminha, G. B., Grillo, C., Rosati, P., et al. 2017, A\&A, 600, A90

Churazov, E., Forman, W., Jones, C., \& Böhringer, H. 2003, ApJ, 590, 225

Clarke, T. E., Blanton, E. L., \& Sarazin, C. L. 2004, ApJ, 616, 178

Colless, M., \& Dunn, A. M. 1996, ApJ, 458, 435

den Hartog, R., \& Katgert, P. 1996, MNRAS, 279, 349

Deshev, B., Finoguenov, A., Verdugo, M., et al. 2017, A\&A, 607, A131

Diaferio, A. 1999, MNRAS, 309, 610

Diaferio, A., \& Geller, M. J. 1997, ApJ, 481, 633

Diaferio, A., Geller, M. J., \& Rines, K. J. 2005, ApJL, 628, L97

Dressler, A., \& Shectman, S. A. 1988, AJ, 95, 985

Dupke, R. A., \& Bregman, J. N. 2001a, ApJ, 562, 266

Dupke, R. A., \& Bregman, J. N. 2001b, ApJ, 547, 705

Dupke, R. A., \& Bregman, J. N. 2006, ApJ, 639, 781

Eckert, D., Gaspari, M., Owers, M. S., et al. 2017, A\&A, 605, A25

Eckert, D., Molendi, S., Owers, M., et al. 2014, A\&A, 570, A119

Einasto, M., Deshev, B., Lietzen, H., et al. 2018, A\&A, 610, A82

Fabricant, D., Fata, R., Roll, J., et al. 2005, PASP, 117, 1411

Feretti, L., Giovannini, G., Govoni, F., \& Murgia, M. 2012, A\&ARv, 20, 54 Geller, M. J., \& Beers, T. C. 1982, PASP, 94, 421

Geller, M. J., Diaferio, A., Rines, K. J., \& Serra, A. L. 2013, ApJ, 764, 58

Geller, M. J., Hwang, H. S., Dell'Antonio, I. P., et al. 2016, ApJS, 224, 11

Geller, M. J., Hwang, H. S., Fabricant, D. G., et al. 2014, ApJS, 213, 35

Girardi, M., Mercurio, A., Balestra, I., et al. 2015, A\&A, 579, A4

Govoni, F., Feretti, L., Giovannini, G., et al. 2001, A\&A, 376, 803

Govoni, F., Ferrari, C., Feretti, L., et al. 2012, A\&A, 545, A74

Grillo, C., Suyu, S. H., Rosati, P., et al. 2015, ApJ, 800, 38

Guennou, L., Adami, C., Durret, F., et al. 2014, A\&A, 561, A112

Gutierrez, K., \& Krawczynski, H. 2005, ApJ, 619, 161

Ho, P. T. P., Altamirano, P., Chang, C.-H., et al. 2009, ApJ, 694, 1610

Hoekstra, H. 2003, MNRAS, 339, 1155

Hwang, H. S., Geller, M. J., Diaferio, A., Rines, K. J., \& Zahid, H. J. 2014, ApJ, 797, 106

Hwang, H. S., \& Lee, M. G. 2007, ApJ, 662, 236

Ichinohe, Y., Simionescu, A., Werner, N., \& Takahashi, T. 2017, MNRAS, 467,3662 
Ivezic, Z., Connolly, A. J., VanderPlas, J. T., \& Gray, A. 2014, Statistics, Data Mining, and Machine Learning in Astronomy: A Practical Python Guide for the Analysis of Survey Data (Princeton, NJ: Princeton Univ. Press)

Jonathan Goodman, J. W. 2010, Communications in Applied Mathematics and Computational Science, 5, 65

Komatsu, E., Smith, K. M., Dunkley, J., et al. 2011, ApJS, 192, 18

Kurtz, M. J., \& Mink, D. J. 1998, PASP, 110, 934

Laganá, T. F., Andrade-Santos, F., \& Lima Neto, G. B. 2010, A\&A, 511, A15

Liu, A., Yu, H., Tozzi, P., \& Zhu, Z.-H. 2015, ApJ, 809, 27

Liu, A., Yu, H., Tozzi, P., \& Zhu, Z.-H. 2016, ApJ, 821, 29

Manolopoulou, M., \& Plionis, M. 2017, MNRAS, 465, 2616

Markevitch, M., Gonzalez, A. H., David, L., et al. 2002, ApJL, 567, L27

Markevitch, M., Govoni, F., Brunetti, G., \& Jerius, D. 2005, ApJ, 627, 733

Markevitch, M., Ponman, T. J., Nulsen, P. E. J., et al. 2000, ApJ, 541, 542

Markevitch, M., \& Vikhlinin, A. 2007, PhR, 443, 1

Markevitch, M., Vikhlinin, A., \& Mazzotta, P. 2001, ApJL, 562, L153

Mazzotta, P., Markevitch, M., Vikhlinin, A., et al. 2001, ApJ, 555, 205

Mink, D. J., Wyatt, W. F., Caldwell, N., et al. 2007, in ASP Conf. Ser. 376, Astronomical Data Analysis Software and Systems XVI, ed. R. A. Shaw, F. Hill, \& D. J. Bell (San Francisco, CA: ASP), 249

Munari, E., Biviano, A., Borgani, S., Murante, G., \& Fabjan, D. 2013, MNRAS, 430, 2638

Munari, E., Biviano, A., \& Mamon, G. A. 2014, A\&A, 566, A68

Navarro, J. F., Frenk, C. S., \& White, S. D. M. 1996, ApJ, 462, 563

Oegerle, W. R., Hill, J. M., \& Fitchett, M. J. 1995, AJ, 110, 32

Okabe, N., Futamase, T., Kajisawa, M., \& Kuroshima, R. 2014, ApJ, 784, 90

Okabe, N., \& Smith, G. P. 2016, MNRAS, 461, 3794

Okabe, N., \& Umetsu, K. 2008, PASJ, 60, 345

Owers, M. S., Nulsen, P. E. J., \& Couch, W. J. 2011, ApJ, 741, 122

Parekh, V., Durret, F., Padmanabh, P., \& Pandge, M. B. 2017, MNRAS, 470,3742
Parekh, V., van der Heyden, K., Ferrari, C., Angus, G., \& Holwerda, B. 2015, A\&A, 575, A127

Pinkney, J., Roettiger, K., Burns, J. O., \& Bird, C. M. 1996, ApJS, 104, 1

Rajpurohit, K., Hoeft, M., van Weeren, R. J., et al. 2018, ApJ, 852, 65

Riseley, C. J., Scaife, A. M. M., Wise, M. W., \& Clarke, A. O. 2017, A\&A, 597, A96

Rossetti, M., Eckert, D., De Grandi, S., et al. 2013, A\&A, 556, A44

Sanders, J. S. 2006, MNRAS, 371, 829

Sanders, J. S., Fabian, A. C., Russell, H. R., Walker, S. A., \& Blundell, K. M. 2016, MNRAS, 460, 1898

Sanders, J. S., Fabian, A. C., \& Taylor, G. B. 2005, MNRAS, 356, 1022

Serna, A., \& Gerbal, D. 1996, A\&A, 309, 65

Serra, A. L., \& Diaferio, A. 2013, ApJ, 768, 116

Serra, A. L., Diaferio, A., Murante, G., \& Borgani, S. 2011, MNRAS, 412, 800

Smith, R. K., Brickhouse, N. S., Liedahl, D. A., \& Raymond, J. C. 2001, ApJL, 556, L91

Song, H., Hwang, H. S., Park, C., \& Tamura, T. 2017, ApJ, 842, 88

Tchernin, C., Eckert, D., Ettori, S., et al. 2016, A\&A, 595, A42

Tittley, E. R., \& Henriksen, M. 2005, ApJ, 618, 227

Umetsu, K., Birkinshaw, M., Liu, G.-C., et al. 2009, ApJ, 694, 1643

Umetsu, K., Medezinski, E., Nonino, M., et al. 2014, ApJ, 795, 163

Venturi, T., Rossetti, M., Brunetti, G., et al. 2017, A\&A, 603, A125

Vikhlinin, A., Kravtsov, A., Forman, W., et al. 2006, ApJ, 640, 691

Wen, Z. L., \& Han, J. L. 2013, MNRAS, 436, 275

Wilms, J., Allen, A., \& McCray, R. 2000, ApJ, 542, 914

Yahil, A., \& Vidal, N. V. 1977, ApJ, 214, 347

Yu, H., Diaferio, A., Agulli, I., Aguerri, J. A. L., \& Tozzi, P. 2016, ApJ, 831,156

Yu, H., Serra, A. L., Diaferio, A., \& Baldi, M. 2015, ApJ, 810, 37

Zarattini, S., Girardi, M., Aguerri, J. A. L., et al. 2016, A\&A, 586, A63 